ACCEPTED MANUSCRIPT

\title{
Van der Waals integration of silicene and hexagonal boron nitride
}

To cite this article before publication: Frank B. Wiggers et al 2019 2D Mater. in press https://doi.org/10.1088/2053-1583/ab0a29

\section{Manuscript version: Accepted Manuscript}

Accepted Manuscript is "the version of the article accepted for publication including all changes made as a result of the peer review process, and which may also include the addition to the article by IOP Publishing of a header, an article ID, a cover sheet and/or an 'Accepted Manuscript' watermark, but excluding any other editing, typesetting or other changes made by IOP Publishing and/or its licensors"

This Accepted Manuscript is @ 2019 IOP Publishing Ltd.

During the embargo period (the 12 month period from the publication of the Version of Record of this article), the Accepted Manuscript is fully protected by copyright and cannot be reused or reposted elsewhere.

As the Version of Record of this article is going to be / has been published on a subscription basis, this Accepted Manuscript is available for reuse under a CC BY-NC-ND 3.0 licence after the 12 month embargo period.

After the embargo period, everyone is permitted to use copy and redistribute this article for non-commercial purposes only, provided that they adhere to all the terms of the licence https://creativecommons.org/licences/by-nc-nd/3.0

Although reasonable endeavours have been taken to obtain all necessary permissions from third parties to include their copyrighted content within this article, their full citation and copyright line may not be present in this Accepted Manuscript version. Before using any content from this article, please refer to the Version of Record on IOPscience once published for full citation and copyright details, as permissions will likely be required. All third party content is fully copyright protected, unless specifically stated otherwise in the figure caption in the Version of Record.

View the article online for updates and enhancements. 


\title{
VAN DER WAALS INTEGRATION OF SILICENE AND HEXAGONAL BORON NITRIDE
}

\author{
F. B. Wiggers ${ }^{1, \$}$, A. Fleurence ${ }^{2}$, K. Aoyagi ${ }^{2}$, T. Yonezawa², Y. Yamada-Takamura ${ }^{2}$, H. Feng ${ }^{3,4}$, J. \\ Zhuang $^{3,4}$, Y. Du ${ }^{3,4}$, A. Y. Kovalgin ${ }^{1}$, and M. P. de Jong ${ }^{1, *}$ \\ ${ }^{1}$ MESA+ Institute for Nanotechnology, University of Twente, 7500 AE Enschede, The Netherlands \\ ${ }^{2}$ School of Materials Science, Japan Advanced Institute of Science and Technology, Nomi, \\ Ishikawa 923-1292, Japan
}

${ }^{3}$ Institute for Superconducting and Electronic Materials, Australian Institute for Innovative Materials, University of Wollongong, Wollongong, New South Wales 2525, Australia

${ }^{4}$ Department of Physics, and BUAA-UOW Joint Research Centre, Beihang University, Beijing, 100191, China

spresent address: ASML Netherlands B.V.; De Run 6501, 5504 DR Veldhoven, The Netherlands

*Correspondence to: M.P.deJong@utwente.nl

\begin{abstract}
Silicene, the silicon analogue of graphene, consists of an atomically buckled honeycomb lattice of silicon atoms. Theory predicts exceptional electronic properties, including Dirac fermions and a topological spin Hall insulator phase. An important obstacle impeding exploration of such properties in electronic devices is the chemical sensitivity of silicene, hampering its incorporation in layer stacks. Here we show experimentally that epitaxial silicene and hexagonal boron nitride (h-BN) can be stacked without perturbing the electronic properties of silicene. Intercalated silicene underneath epitaxial h-BN on $\mathrm{ZrB}_{2}(0001)$ substrate films is obtained by depositing $\mathrm{Si}$ atoms at room temperature. Using (angle resolved) photoelectron spectroscopy (ARPES, PES) and scanning tunneling microscopy (STM) we find that the intercalated silicene exhibits the same electronic properties as epitaxial silicene on $\mathrm{ZrB}_{2}$, while it resists oxidation in air up to several hours. This is an essential step towards the development of layer stacks that allow for fabrication of devices.
\end{abstract}




\section{Introduction}

The buckled nature of silicene, ${ }^{1,2}$ the silicon analogue of graphene, ${ }^{3}$ is predicted to allow for electrostatic tuning of its electronic properties across a range of behaviors, including that of a topological insulator. ${ }^{4}$ However, silicene is chemically reactive and oxidizes upon exposure to air. While this property can be favorably exploited for chemical surface modification, it hinders ex situ characterization and the integration of silicene in an electronic device. This can be solved by an insulating encapsulation layer, functioning as both a protective layer and a gate dielectric layer. Epitaxial $\mathrm{ZrB}_{2}$ thin films grown on $\mathrm{Si}(111)$ substrates have the unique property to spontaneously form a layer of epitaxial silicene on their surfaces upon annealing. ${ }^{5}$ In addition, the $\mathrm{ZrB}_{2}$ surface can also be terminated with an epitaxial hexagonal boron nitride ( $\mathrm{h}$-BN) monolayer by plasma nitridation and subsequent annealing at $900{ }^{\circ} \mathrm{C} .{ }^{6}$ Hexagonal $\mathrm{BN}$ is an inert, wide-bandgap semiconductor and is as such a suitable template as well as an encapsulation layer. Further, theoretical simulations studying silicene on or between h-BN layers found that silicene is stable, interacts weakly with $\mathrm{h}-\mathrm{BN}$, and retains the electronic properties of free-standing silicene. ${ }^{7-14}$ This combination of experimental findings and theoretical predictions motivated us to explore the vertical integration of epitaxial silicene with $\mathrm{h}-\mathrm{BN}$ on the $\mathrm{ZrB}_{2}(0001)$ surface.
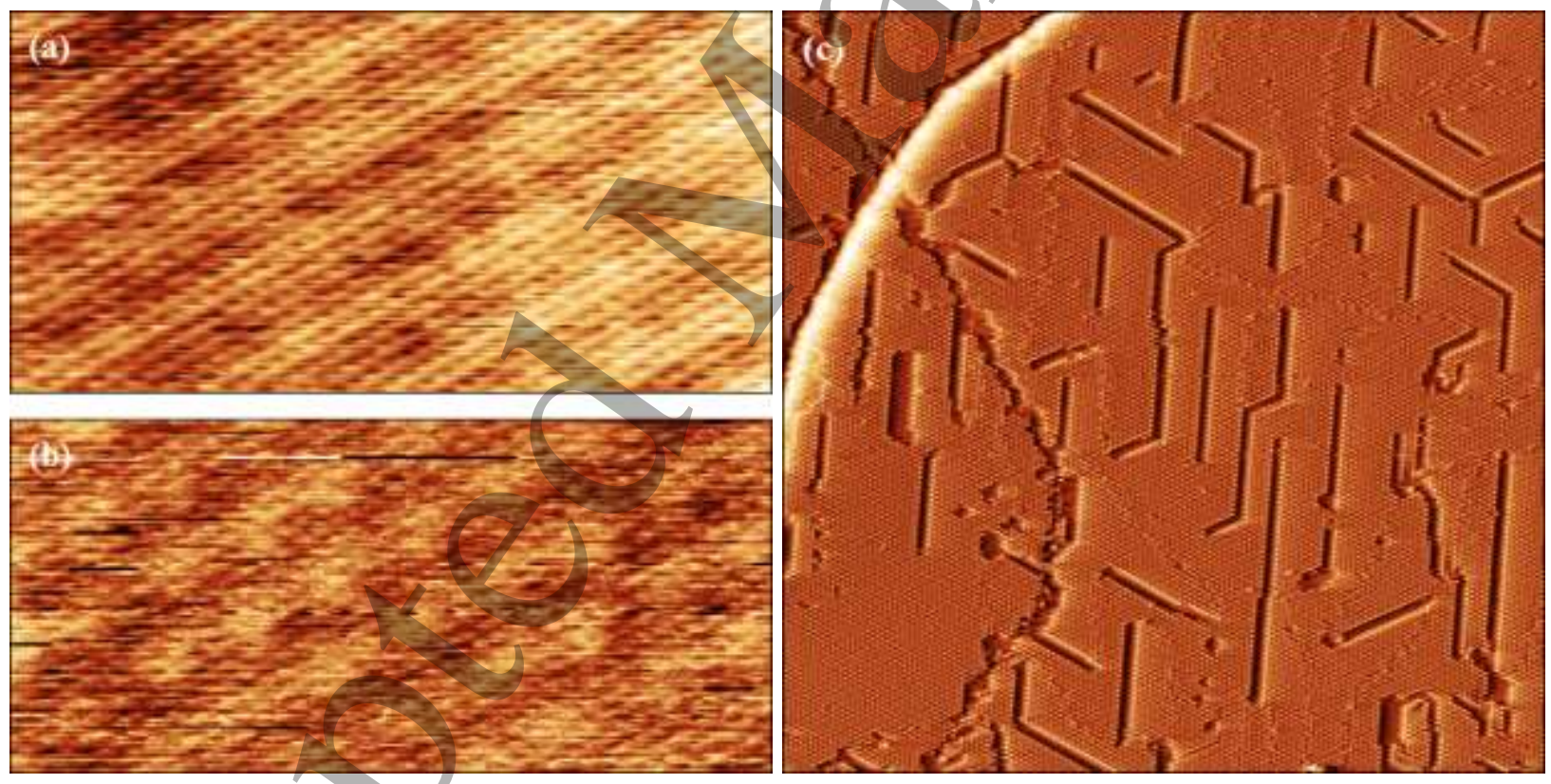

Figure 1: (a) $(10 \mathrm{~nm} \times 5 \mathrm{~nm}, \mathrm{~V}=80 \mathrm{mV}, \mathrm{I}=1.2 \mathrm{nA}$, room temperature $) \mathrm{STM}$ images of a pristine $\mathrm{h}$ BN-terminated $\mathrm{ZrB}_{2}$ surface showing the $(1 \times 1)$-h-BN lattice, (b) $(10 \mathrm{~nm} \times 5 \mathrm{~nm}, \mathrm{~V}=0.1 \mathrm{~V}, \mathrm{I}=1 \mathrm{nA}$, room temperature) the $\mathrm{h}-\mathrm{BN} / \mathrm{ZrB}_{2}$ moiré pattern, depending on the sample bias voltage, and (c) (95 nm $\times 95 \mathrm{~nm}, \mathrm{~V}=-1.1 \mathrm{~V}, \mathrm{I}=0.4 \mathrm{nA}$, room temperature) a differentiated STM image after deposition of $4 \mathrm{ML}$ of $\mathrm{Si}$, resulting in near-complete coverage by a $2 \mathrm{D}$ Si layer. 


\section{Results and discussion}

The as-annealed $\mathrm{h}-\mathrm{BN}$ terminated $\mathrm{ZrB}_{2}$ surface (hereafter referred to as pristine) exhibits a short periodic corrugation of $2.5 \AA$ corresponding to the $(1 \times 1)$ unit cell of $h-B N$ and a periodicity of 11.7 $\AA ̊ n$ corresponding to a moiré pattern, as shown in Fig. 1 . The moire pattern is the result of the overlay of the $\mathrm{h}-\mathrm{BN}$ lattice on $\mathrm{ZrB}_{2}(0001)$, the latter having a lattice constant of $3.186 \AA .{ }^{15} \mathrm{lt}$ follows that 5 unit cells of $h-B N$ are almost commensurate with 4 unit cells of $\mathrm{ZrB}_{2}$. The pristine surface also contains some residual $\mathrm{Si}$, mostly residing in crystalline $2 \mathrm{D}$ islands as observed by STM (see Fig. S1), with lattice periodicity and crystal orientation identical to that of the $(\sqrt{ } 3 \times \sqrt{ } 3)$ reconstructed silicene layer on $\mathrm{ZrB}_{2}$ without $\mathrm{h}-\mathrm{BN}$. The $(1 \times 1)$ lattice constant of the latter was previously reported as $3.65 \AA .{ }^{5}$

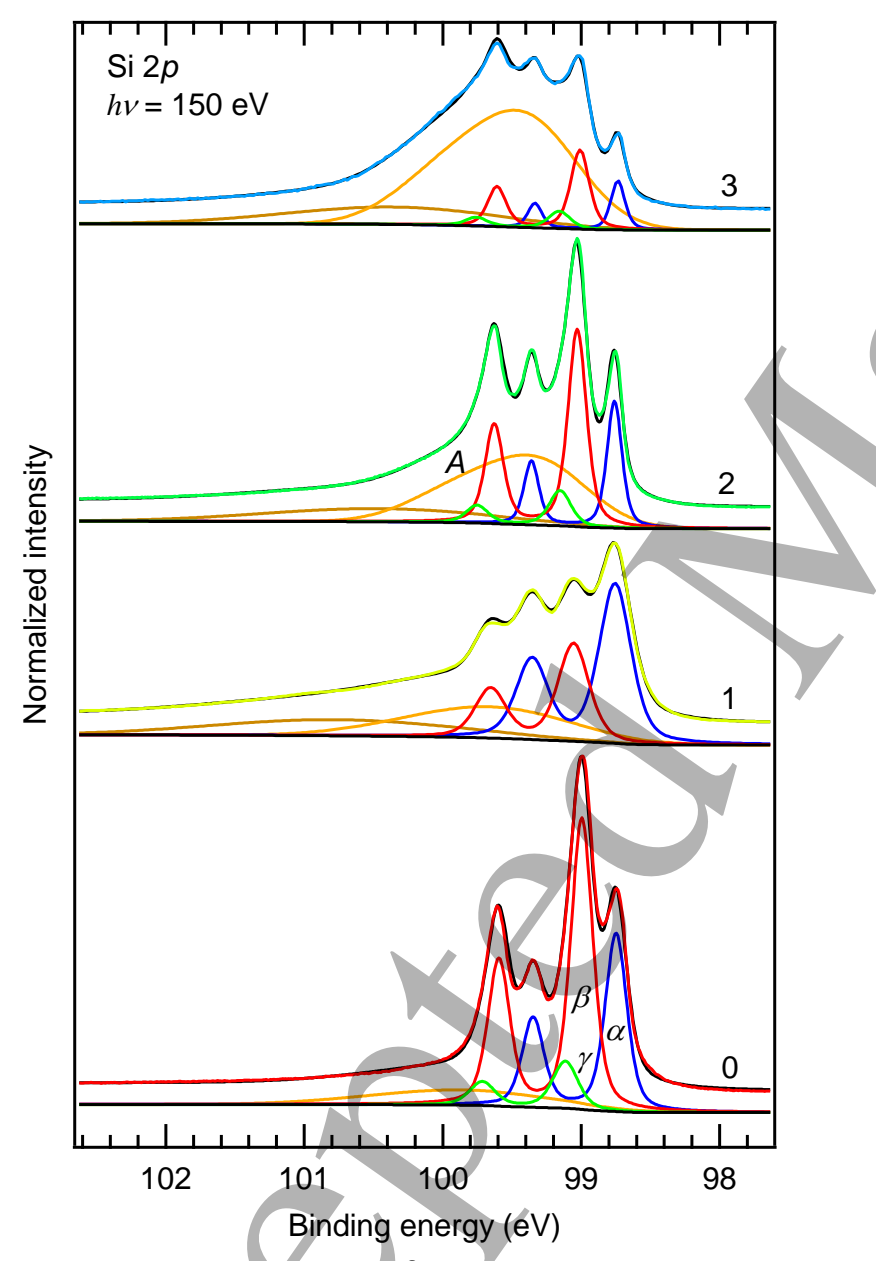

Figure 2: PES spectra of the Si $2 p$ core-level region before and after deposition of Si on the h-BNterminated $\mathrm{ZrB}_{2}$ surface. The spectra are presented in chronological order: (1) pristine h-BNterminated $\mathrm{ZrB}_{2}$ surface, (2) after deposition of $1.6 \mathrm{ML}$ of $\mathrm{Si}$, (3) after deposition of an additional 3.2 ML of Si. Spectrum 0 corresponds to epitaxial silicene on $\mathrm{ZrB}_{2}$ without $\mathrm{h}-\mathrm{BN}$, included here for comparison. The Si $2 p$ spectra are shown together with peak-fitting results. The sum of the fitted data is plotted behind the measured data, while the individual components and the background are offset. 
The Si $2 p$ spectrum of the pristine surface, shown in Fig. 2 (spectrum 1 ), shows two clearly resolved doublets and a tail. The sharp peaks are attributed to the crystalline 2D Si islands. The Si $2 p_{3 / 2}$ peaks of the two doublets are at a binding energy of $99.05 \mathrm{eV}$ and $98.75 \mathrm{eV}$, where the latter doublet has a higher intensity.

Upon deposition of Si on the surface at room temperature, the 2D Si islands increase in number and size, while their lattice periodicity and crystal orientation remain unchanged. Although the surface coverage by the Si islands increases and nearly reaches full coverage, as shown in Fig. 1(c) for a deposition of $4 \mathrm{ML}$ of Si (see also Fig. S2), no nucleation of additional layers on top of this layer, which would yield a stepped surface, was observed by STM. The deposited Si that is not incorporated into the 2D Si layer most likely forms three-dimensional (3D) disordered structures on the surface, or is incorporated into pre-existing ones (see Fig. S3). The 2D Si layer shown in Fig. 1 (c) exhibits three types of defects: holes ranging in size from one to few tens of unit cells, trenches, and anti-phase boundaries (see Fig. S2). Trenches of a few ( $33 \times \sqrt{ } 3)$-cells wide and oriented along crystallographic axes remain in the 2D Si layer regardless of Si surface coverage, most probably to relieve strain. As we show below, the 2D Si layer is formed by intercalation of $\mathrm{Si}$ atoms underneath $\mathrm{h}-\mathrm{BN}$, such that a competition between h-BN interacting with $\mathrm{ZrB}_{2}$ and $\mathrm{h}-\mathrm{BN}$ accommodating the intercalated Si might play a role as well. We expect that defects in the $h-B N$ layer facilitate the intercalation process. Here "defects" refers to any kind of imperfection in the epitaxial h-BN layer, including point defects, domain boundaries, and "open" spaces where we have silicene termination instead.

After deposition of $\mathrm{Si}$, the Si $2 p$ spectrum shows a significant change (see Supporting Information for changes to core levels of other elements), as shown in Fig. 3 (spectrum 2, $1.6 \mathrm{ML}$ of Si deposited). Two sharp doublets are again resolved, but their intensity ratio is now such that the doublet at higher binding energy is most intense. The spectrum appears to be very similar to the Si $2 p$ spectrum of epitaxial silicene on the $\mathrm{ZrB}_{2}$ surface without h-BN, shown in Fig. 2 (spectrum 0 ), which has been previously studied. ${ }^{5,16-18}$ This is confirmed by least-squares peak fitting (see Methods for details), which shows that the sharp Si $2 p$ features can be fitted with $\alpha, b$, and $\gamma$ components with similar binding energies and area ratios: $98.75 \mathrm{eV}, 98.99 \mathrm{eV}$, and $99.11 \mathrm{eV}$ with area ratios of 1.8:3.0:0.6, respectively, for epitaxial silicene layer on $\mathrm{ZrB}_{2}$, and $98.76 \mathrm{eV}, 99.03 \mathrm{eV}$, and $99.15 \mathrm{eV}$, with area ratios 1.6:3.0:0.6 for 1.6 ML of $\mathrm{Si}$ on the h-BN-terminated $\mathrm{ZrB}_{2}$. This shows that the corresponding $\mathrm{Si}$ atoms in both 2D layers are in essentially the same chemical environment. This is strong eyidence for the intercalation of deposited Si underneath the $\mathrm{h}-\mathrm{BN}$ layer, where it forms epitaxial silicene on the $\mathrm{ZrB}_{2}$ surface. ${ }^{16}$ The broad component $A$ is attributed to disordered $\mathrm{Si}$ on top of the h-BN-terminated surface. Upon deposition of a total of $4.8 \mathrm{ML}$ of $\mathrm{Si}$, this broad contribution becomes the dominant feature of the spectrum, as shown in Fig. 2 (spectrum 3), while the two sharp doublets can still be resolved (fitted binding energies of the $\alpha$, $b$, and $\gamma$ components are $98.73 \mathrm{eV}, 99.01 \mathrm{eV}$, and $99.16 \mathrm{eV}$, and their area ratios are 1.4:3.0:0.8). The increase of component $A$ relative to the silicene-related peaks suggests that the intercalation of Si has saturated, leaving most of the deposited Si in a disordered phase on the surface. It is noted that all Si $2 p$ spectra, shown in Fig. 2, contain broad contributions at higher binding 
energies, attributed to oxidized or disordered $\mathrm{Si}$. A comparison of the $\mathrm{Si} 2 p$ spectra after $\mathrm{Si}$ deposition with that of the pristine h-BN-terminated $\mathrm{ZrB}_{2}$ surface, which showed a different spectral line shape, is discussed in the Supporting Information.

We now demonstrate that the h-BN layer does not affect the electronic properties of silicene, based upon the valence electronic structures of pristine $\mathrm{h}-\mathrm{BN}$ terminated $\mathrm{ZrB}_{2}$ and intercalated silicene, characterized by ARPES. The same notation used in previous studies of the low bindingenergy region of silicene on $\mathrm{ZrB}_{2}$ is used to mark the individual features in the ARPES spectra. ${ }^{5,18-}$ ${ }^{20}$ The ARPES spectrum, measured along the $\Gamma-\mathrm{M}-\Gamma$ direction of $\mathrm{ZrB}_{2}$, of the pristine $\mathrm{h}-\mathrm{BN} / \mathrm{ZrB} 2$ surface is shown in Fig. 3(a). The spectrum was measured using a photon energy of $50 \mathrm{eV}$. It features band $\mathrm{S}_{2}$ (see Fig. $3(\mathrm{c})$ ), associated with $\mathrm{ZrB}_{2}$ surface states. Features associated with the $\pi$-electronic bands of epitaxial silicene are not observed, suggesting that there is either little or no silicene at all on the surface.

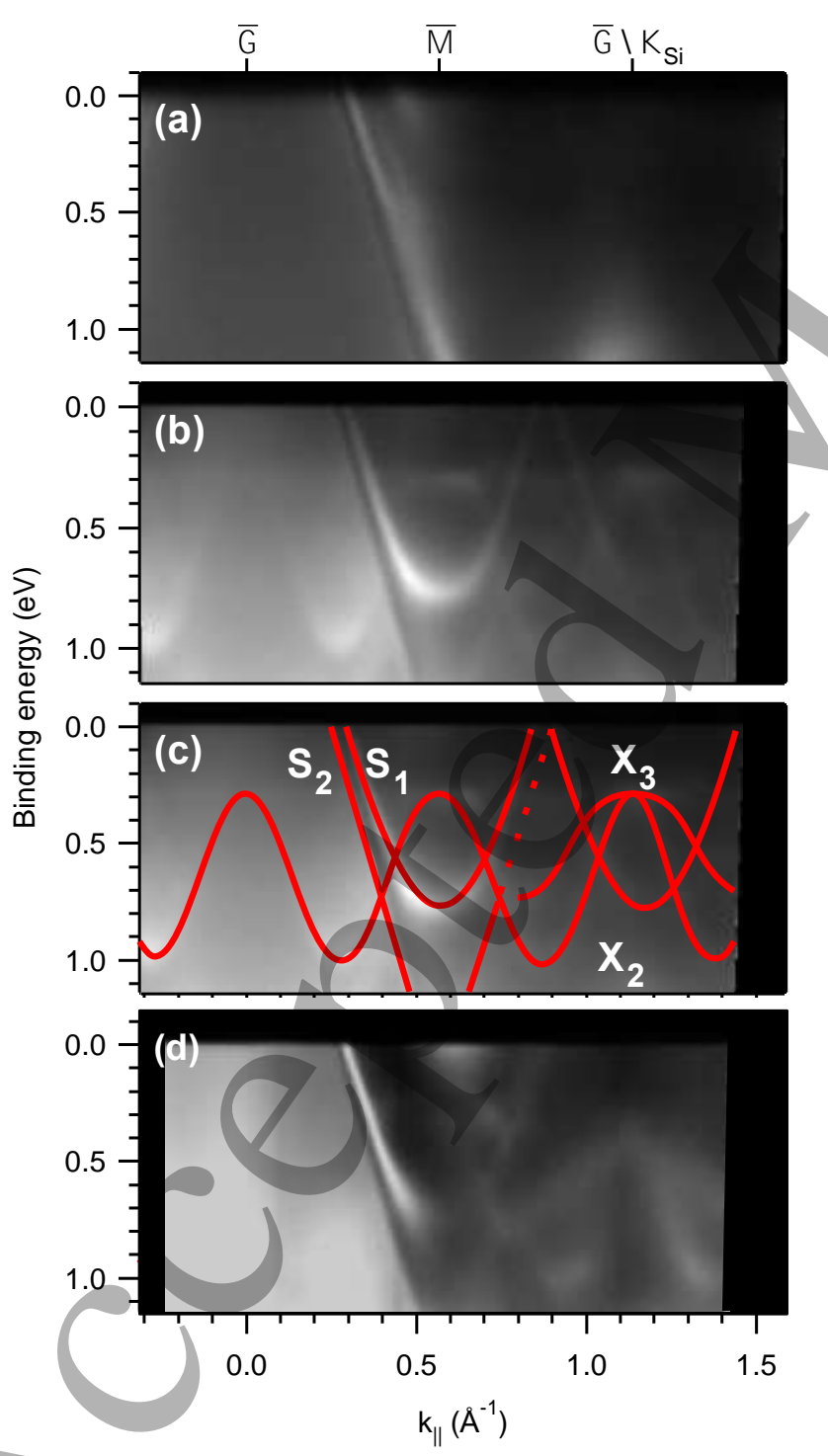


Figure 3: ARPES spectra of (a) pristine h-BN-terminated $\mathrm{ZrB}_{2}$ measured with $50 \mathrm{eV}$ photon energy, and $(\mathrm{b}-\mathrm{c})$ after deposition of $1.6 \mathrm{ML}$ of Si measured with $43 \mathrm{eV}$ photon energy, sample temperature $10 \mathrm{~K}$. (d) ARPES spectrum of silicene on $\mathrm{ZrB}_{2}$ measured with $45 \mathrm{eV}$ photon energy and sample temperature $22 \mathrm{~K}$.

The ARPES spectrum of the $\mathrm{h}-\mathrm{BN} / \mathrm{ZrB}_{2}$ surface with 1.6 ML of Si deposited is shown in Figs. 3(b-c), and is also measured along the $\Gamma-\mathrm{M}-\Gamma$ direction of $\mathrm{ZrB}_{2}$. The spectrum is measured with a photon energy of $43 \mathrm{eV}$. It shows $\mathrm{ZrB}_{2}$-derived bands $\mathrm{S}_{1}$ and $\mathrm{S}_{2}$. In addition, the silicene-related bands $\mathrm{X}_{2}$ and $X_{3}$ are now observed. The band structure is in good agreement with that of epitaxial silicene on $\mathrm{ZrB}_{2}$ without an h-BN layer, ${ }^{5,18,20}$ shown in Fig. 3(d). This can be explained by the intercalation of the deposited Si underneath the h-BN monolayer, where it forms epitaxial silicene on the $\mathrm{ZrB}_{2}$ surface. Importantly, the close similarity of the band structures indicates that the silicene exhibits negligible hybridization with the h-BN on top.

In order to determine the effectiveness of encapsulation by the $\mathrm{h}-\mathrm{BN}$ monolayer, intercalated silicene, formed by deposition of $0.7 \mathrm{ML}$ of $\mathrm{Si}$ on an h-BN-terminated $\mathrm{ZrB}_{2}(0001)$ surface at room temperature, was exposed to air at atmospheric pressure in the loadlock (vented with $\mathrm{N}_{2}$ gas, and subsequently opened for 10 seconds) for 5 minutes. For the purpose of comparison, spontaneously-formed silicene ${ }^{5}$ and single-domain silicene ${ }^{18}$ on $\mathrm{ZrB}_{2}(0001)$ thin films, grown on $\mathrm{Si}(111)$ substrates, were also exposed to air for 5 minutes in the same way. Si $2 p$ spectra of these samples are shown in Fig. 4(a). 

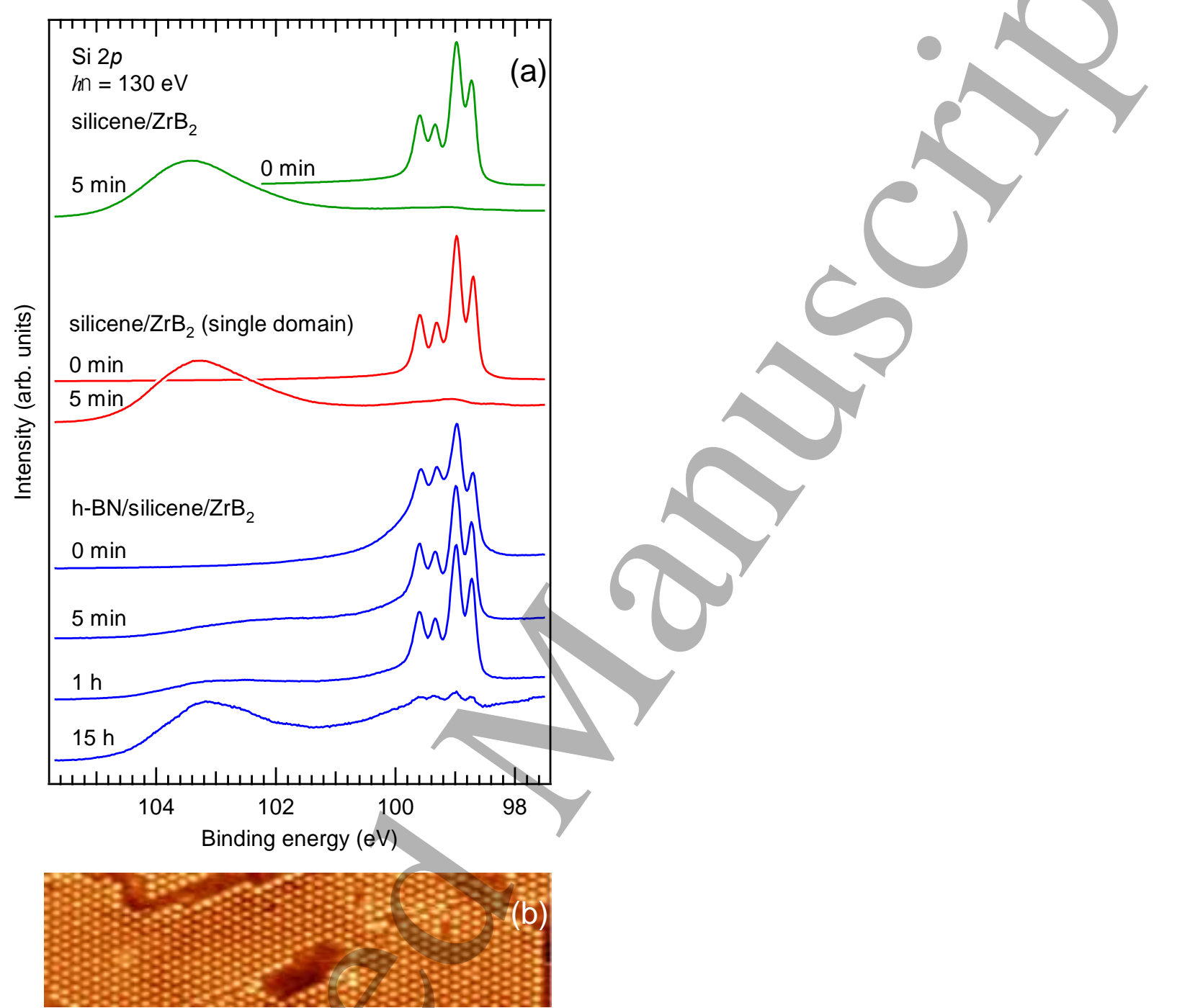

Figure 4: (a) PES spectra of the Si $2 p$ core-level region of spontaneously-formed silicene on $\mathrm{ZrB}_{2}$, single-domain silicene on $\mathrm{ZrB}_{2}$, and intercalated silicene on $\mathrm{h}$-BN-terminated $\mathrm{ZrB}_{2}$, before and after exposure to air. It is noted that the intensities of the spectra have been individually rescaled. The binding energy scale has been calibrated using the $B$ component of silicene on $\mathrm{ZrB}_{2}$. (b) $(8 \mathrm{x}$ $30 \mathrm{~nm}^{2}, \mathrm{~V}=-700 \mathrm{mV}, \mathrm{l}=100 \mathrm{pA}$, room temperature) STM image of intercalated silicene on $\mathrm{h}-\mathrm{BN}$ terminated $\mathrm{ZrB}_{2}$, recorded after exposing the sample to air for 5 minutes.

Exposure of the intercalated silicene layer to air for 5 minutes results in the appearance of a broad component centered at $102 \mathrm{eV}$, attributed to Si sub-oxide. ${ }^{21}$ Interestingly, the silicene spectrum has become sharper. This can be explained by the preferential oxidation of the disordered Si (which most probably has not intercalated), which moved the associated spectral intensity towards higher binding energies. Furthermore, when spontaneously-formed silicene or single- 
domain silicene was exposed to air for 5 minutes, as shown in Fig. 4(a), all silicene-related intensities disappeared, and a broad component appeared at $103.3 \mathrm{eV}$, attributed to $\mathrm{SiO}_{2} .{ }^{21}$ This suggests that both non-encapsulated silicene layers became fully oxidized. In comparison, the spectrum of the silicene layer encapsulated by h-BN shows relatively little Si oxide, even after prolonged air exposure for 1 hour. This provides evidence for the encapsulation of the silicene layer and its effectiveness, as is also demonstrated by the STM measurement of an air-exposed sample shown in Fig. 4(b), which still exhibits atomic resolution. Exposing the encapsulated silicene layer again to air for 15 hours in total (by storing the sample in air outside the loadlock) results in the near-complete disappearance of the silicene spectrum and the appearance of a broad component at $103.2 \mathrm{eV}$, also observed for the non-encapsulated silicene layers after just 5 minutes, suggesting that most of the silicene layer has become oxidized. This demonstrates a limit to the effectiveness of the h-BN encapsulation layer, which nevertheless allows for enough time (at least 1 hour) for ex situ sample transfer, characterization, or processing.

\section{Conclusions}

In conclusion, we find that the deposition of $\mathrm{Si}$ atoms onto a monolayer of $\mathrm{h}-\mathrm{BN}$ on a $\mathrm{ZrB}_{2}$ thin film at room temperature leads to the formation of an intercalated, self-terminating, epitaxial silicene layer underneath the h-BN monolayer. From an electronic structure perspective, there is no indication of interaction between the h-BN layer and the silicene layer, demonstrating vertical integration of $\mathrm{h}-\mathrm{BN}$ and silicene without perturbing the electronic properties of the latter. However, the large-scale domain structure of silicene is affected, characterized by islands separated by trenches. The intercalated silicene layer also exhibits edges which corresponds to armchair edges of the silicene honeycomb lattice, in contrast to the continuous sheet of silicene on $\mathrm{ZrB}_{2}$ without h-BN. Importantly, the h-BN encapsulation layer hinders the oxidation of the silicene layer underneath and enables ex situ sample transfer, characterization, or processing.

\section{Methods}

Sample preparation and STM measurements took place at JAIST (Japan). ZrB ${ }_{2}$ thin films are grown on $\mathrm{Si}(111)$ substrates by ultra-high vacuum chemical vapor epitaxy (UHV-CVE), which is described elsewhere. ${ }^{15}$ It was previously found that the surface of the $\mathrm{ZrB}_{2}$ thin film is terminated with a spontaneously-formed monolayer of silicene upon annealing at $800^{\circ} \mathrm{C}$ in UHV, with the Si atoms originating from the Si substrate. ${ }^{5}$ Subsequently, in a separate UHV system, an h-BN monolayer is formed by nitridation of the surface using a nitrogen plasma and a subsequent annealing treatment at $900{ }^{\circ} \mathrm{C}$ in UHV, as reported in previous work. ${ }^{6}$ This system consists of a molecular beam epitaxy chamber equipped with an RF plasma source, a chamber with a PES system (base pressure of $1 \times 10^{-10} \mathrm{mbar}$ ), and a chamber with an STM system (base pressure of $1 \times 10^{-10} \mathrm{mbar}$ ). $\mathrm{Si}$ deposition was carried out by exposing the h-BN surface, held at room temperature, to the Si flux from the deposition source made of a narrow Si strip that is resistively heated by directcurrent, during which the pressure in the chamber was $3 \times 10^{-10} \mathrm{mbar}$. It is noted that during deposition the sample is slightly heated by radiative heating from the $\mathrm{Si}$ deposition source, such 
that the substrate temperature is somewhat higher than room temperature. STM measurements were performed in constant-current mode and at room temperature using a Pt-Ir tip. The Si deposition rate was calibrated by depositing $\mathrm{Si}$ on a Si(111)-(7×7) surface and measuring the coverage of epitaxial growth using STM. The deposition rate of the Si source was 1 monolayer $(\mathrm{ML})$, defined as 1 bilayer of $\mathrm{Si}(111)$, per 30 minutes.

Additional STM measurements were performed at the STM lab at ISEM (University of Wollongong, Australia). The system consists of three interconnected UHV chambers: a loadlock, preparation chamber, and analysis chamber. The preparation chamber $\left(1 \times 10^{-10} \mathrm{mbar}\right.$ base pressure) was used to anneal the sample by e-beam heating, and also has a Si source made of a narrow Si strip that is resistively heated. The deposition rate was $1 \mathrm{ML}$ of Si per 30 minutes. The analysis chamber $\left(6 \times 10^{-11} \mathrm{mbar}\right.$ base pressure) contains the STM system. The STM measurements were performed in constant-current mode using a Pt-Ir tip and a sample temperature of $77 \mathrm{~K}$.

PES and ARPES measurements were recorded at two synchrotron facilities: beamline 105 at Diamond Light Source (UK) and beamline BL6U at UVSOR (Japan). The end station at the HR-ARPES branch of beamline 105 at Diamond Light Source consists of four interconnected chambers: a loadlock, a preparation chamber, and an upper and lower chamber. Another UHV system could be accessed through the preparation chamber to reach a Si deposition source $\left(3 \times 10^{-10} \mathrm{mbar}\right.$ during deposition), consisting of an e-beam heated Si rod, that was calibrated using the $(5 \times 2)$ LEED pattern of Si on $\mathrm{Ag}(110) .{ }^{22}$ The deposition rate was $0.8 \mathrm{ML}$ of Si per 5 minutes. The upper chamber also connects to the preparation chamber, and features a LEED system and access to the lower chamber $\left(2 \times 10^{-10}\right.$ mbar base pressure) for (AR)PES measurements. During measurements the sample was always at a temperature of $10 \mathrm{~K}$. The end station of BL6U at UVSOR consists of three interconnected UHV chambers: a loadlock, a preparation chamber, and an analysis chamber. The preparation chamber $\left(3 \times 10^{-10}\right.$ mbar base pressure $)$ features the $\mathrm{Si}$ deposition source made of a narrow Si strip that is resistively heated. The deposition rate was 1 $M L$ of Si per 5 minutes. The analysis chamber $\left(8 \times 10^{-11} \mathrm{mbar}\right.$ base pressure) features a LEED system and a detector and photon source to perform (AR)PES measurements with the samples at a temperature of $15 \mathrm{~K}$. The binding energy scales of the Si $2 p$ spectra were calibrated with respect to the Fermi level by measuring both the Si $2 p$ core level and the Fermi edge with a photon energy of $150 \mathrm{eV}$. The Zr 3d and B 1s, and N 1s core levels (see Supporting Information) were calibrated by measuring them together with the Si $2 p$ core level with a photon energy of $240 \mathrm{eV}$ and $500 \mathrm{eV}$, respectively.

Least-squares peak fitting of Si $2 p$ core level spectra was performed using a Shirley background and symmetric line shapes, with each component consisting of a doublet with an area ratio of $1: 2$ for the Si $2 p_{1 / 2}$ and Si $2 p_{3 / 2}$ peaks and $0.60 \mathrm{eV}$ spin-orbit splitting. The epitaxial silicene layer on $\mathrm{ZrB}_{2}$ has a honeycomb lattice with three unique atomic sites that each contribute a Si $2 p$ doublet to the $\mathrm{Si} 2 p$ spectrum. The three atomic sites are referred to as $A, B$, and $C$ and occur in the ratio of 2:3:1 with corresponding Si $2 p_{3 / 2}$ core levels $\alpha, b$, and $\gamma \cdot{ }^{16}$ The three components $\alpha, b$, and $\gamma$ associated with silicene, and the two components associated with the 2D Si islands, are fitted with a line shape based on a Gaussian-Lorentzian sum function with a $40-50 \%$ Lorentzian 
character. The other components, e.g. tail features that can be explained by Si sub-oxides that remained after the annealing treatments to remove native Si oxide from the sample surfaces, are Gaussian line shape.

\section{Acknowledgements}

This work is part of the research program of the Foundation for Fundamental Research on Matter (FOM, Grant No. 12PR3054), which is part of the Netherlands Organization for Scientific Research (NWO), and is supported by Joint Research by Institute for Molecular Science (IMS) (IMS program No,206). AF acknowledges support from JSPS KAKENHI Grant Number JP26790005. YYT acknowledges support from JSPS KAKENHI Grant Number JP26246002. HF, JZ and YD acknowledge support from the Australian Research Council through two Discovery Projects (DP160102627 and DP170101467). We thank Diamond Light Source (UK) and experimental support from Dr. Moritz Hoesch, as well as UVSOR (JP) and experimental support from Dr. Hiroyuki Yamane, for having provided synchrotron beam time.

\section{Associated content}

The Supporting Information is available free of charge on ...

\section{References}

1. Takeda, K.; Shiraishi, K. Phys. Rev. B 1994, 50, 14916-14922.

2. Guzman-Verri, G. G.; Voon, L. C. L. Y. Phys. Rev. B 2007, 76, 075131.

3. Novoselov, K. S.; Geim, A. K.; Morozov, S. V.; Jiang, D.; Zhang, Y.; Dubonos, S. V.; Grigorieva, I. V.; Firsov, A. A. Science 2004, 306, 666-669.

4. Ezawa, M. New J. Phys. 2012, 14, 033003.

5. $\quad$ Fleurence, A.; Friedlein, R.; Ozaki, T.; Kawai, H.; Wang, Y.; Yamada-Takamura, Y. Phys. Rev. Lett. 2012, 108, 245501.

6. Wang, Z.-T.; Yamada-Takamura, Y.; Fujikawa, Y.; Sakurai, T.; Xue, Q. K.; Tolle, J.; Kouvetakis, J.; Tsong, I. S. T. J. Appl. Phys. 2006, 100, 033506.

7. Ni, Z.; Liu, Q.; Tang, K.; Zheng, J.; Zhou, J.; Qin, R.; Gao, Z.; Yu, D.; Lu, J. Nano Lett. 2012, 12, 113-118.

8. Liu, H. S.; Gao, J. F.; Zhao, J. J. J. Phys. Chem. C 2017, 117, 10353-10359.

9. Guo, Z. X.; Furuya, S.; Iwata, J.; Oshiyama, A. Phys. Rev. B 2013, 87, 235435.

10. Li, L. Y.; Wang, X. P.; Zhao, X. Y.; Zhao, M. W. Phys. Lett. A 2013, 377, 2628-2632.

11. Kaloni, T. P.; Tahir, M.; Schwingenschlogl, U. Sci. Rep. 2013, 3, 3192.

12. Gao, N.; Li, J. C.; Jiang, Q. Chem. Phys. Lett. 2014, 592, 222-226.

13. Kanno, M.; Arafune, R.; Lin, C. L.; Minamitani, E.; Kawai, M.; Takagi, N. New J. Phys. 2014, 16, 105019.

14. Cai, Y.; Pei, Q.-X.; Zhang, G.; Zhang, Y.-W. J. Appl. Phys. 2016, 119, 065102.

15. Yamada-Takamura, Y.; Bussolotti, F.; Fleurence, A.; Bera, S.; Friedlein, R. Appl. Phys. Lett. 2010, 97, 073109.

16. Lee, C.-C.; Yoshinobu, J.; Mukai, K.; Yoshimoto, S.; Ueda, H.; Friedlein, R.; Fleurence, A.; YamadaTakamura, Y.; Ozaki, T. Phys. Rev. B 2017, 95, 115437.

17. Friedlein, R.; Fleurence, A.; Aoyagi, K.; de Jong, M. P.; Van Bui, H.; Wiggers, F. B.; Yoshimoto, S.; Koitaya, T.; Shimizu, S.; Noritake, H.; Mukai, K.; Yoshinobu, J.; Yamada-Takamura, Y. J. Chem. Phys. 2014, 140, 184704.

18. Fleurence, A.; Gill, T. G.; Friedlein, R.; Sadowski, J. T.; Aoyagi, K.; Copel, M.; Tromp, R. M.; Hirjibehedin, C. F.; Yamada-Takamura, Y. Appl. Phys. Lett. 2016, 108, 151902.

19. Friedlein, R.; Fleurence, A.; Sadowski, J. T.; Yamada-Takamura, Y. Appl. Phys. Lett. 2013, 102, 221603. 
20. Lee, C.-C.; Fleurence, A.; Yamada-Takamura, Y.; Ozaki, T.; Friedlein, R. Phys. Rev. B 2014, 90, 075422.

21. Lu, Z. H.; Graham, M. J.; Jiang, D. T.; Tan, K. H. Appl. Phys. Lett. 1993, 63, 2941-2943.

22. Colonna, S.; Serrano, G.; Gori, P.; Cricenti, A.; Ronci, F. J. Phys.-Cond. Matter 2013, 25, 315301. 